\title{
New GGOS JWG3 on Improved understanding of space weather events and their monitoring
}

\section{Other Conference Item}

\section{Author(s):}

Garcia-Rigo, Alberto; Soja, Benedikt (D)

Publication date:

2020-05

Permanent link:

https://doi.org/10.3929/ethz-b-000459626

Rights / license:

Creative Commons Attribution 4.0 International

Originally published in:

EGUsphere, https://doi.org/10.5194/egusphere-egu2020-20492 
EGU2020-20492

https://doi.org/10.5194/egusphere-egu2020-20492

EGU General Assembly 2020

(c) Author(s) 2021. This work is distributed under

the Creative Commons Attribution 4.0 License.

\title{
New GGOS JWG3 on Improved understanding of space weather events and their monitoring
}

\author{
Alberto Garcia-Rigo ${ }^{1,2}$ and Benedikt Soja ${ }^{3}$ \\ 1UPC-IonSAT, Technical University of Catalonia BarcelonaTech, Barcelona, Spain (alberto.garcia.rigo@upc.edu) \\ ${ }^{2}$ IEEC, Institut d'Estudis Espacials de Catalunya, Barcelona, Spain \\ ${ }^{3}$ Jet Propulsion Laboratory, California Institute of Technology, Pasadena, U.S.A.
}

Multiple space geodetic techniques are capable of measuring effects caused by space weather events. In particular, space weather events can cause ionospheric disturbances correlated with variations in the vertical total electron content (VTEC) or the electron density $(\mathrm{Ne})$ of the ionosphere.

In this regard and in the context of the new Focus Area on Geodetic Space Weather Research within IAG's GGOS (International Association of Geodesy; Global Geodetic Observing System), the Joint Working Group 3 on Improved understanding of space weather events and their monitoring by satellite missions has been created as part of IAG Commission 4, Sub-Commission 4.3 to run for the next four years.

Within JWG3, we expect investigating different approaches to monitor space weather events using the data from different space geodetic techniques and, in particular, combinations thereof. Simulations will be beneficial to identify the contribution of different techniques and prepare for the analysis of real data. Different strategies for the combination of data will also be investigated, in particular, the weighting of estimates from different techniques in order to increase the performance and reliability of the combined estimates. Furthermore, existing algorithms for the detection and prediction of space weather events will be explored and improved to the extent possible. Furthermore, the geodetic measurement of the ionospheric electron density will be complemented by direct observations from the Sun gathered from existing spacecraft, such as SOHO, ACE, SDO, Parker Solar Probe, among others. The combination and joint evaluation of multiple datasets with the measurements of space geodetic observation techniques (e.g. geodetic VLBI) is still a great challenge. In addition, other indications for solar activity - such as the F10.7 index on solar radio flux, SOLERA as EUV proxy or rate of Global Electron Content (dGEC)-, provide additional opportunities for comparisons and validation.

Through these investigations, we will identify the key parameters useful to improve realtime/prediction of ionospheric/plasmaspheric VTEC, Ne estimates, as well as ionospheric perturbations, in case of extreme solar weather conditions. In general, we will gain a better understanding of space weather events and their effect on Earth's atmosphere and near-Earth environment. 